\title{
Infraestructura \\ científica-tecnológica \\ en Chile: propuestas y desafíos
}

Alejandro Jiménez-Montecinos

Profesor de Innovación. Facultad de Economía y Negocios, UAH

La infraestructura científica es clave para sostener las capacidades de I+D de los países. Es la base para adquirir nuevas ventajas competitivas y avanzar hacia una productividad basada en la innovación. Lamentablemente, en Chile hay un déficit en infraestructura y equipamiento para el desarrollo de las ciencias experimentales. El artículo plantea priorizar las inversiones en nuevos espacios para la I+D y propone una nueva infraestructura -separada de la institucionalidad universitaria- orientada a atender las necesidades de la industria y la generación de empresas de base científica.

CONTEXTO

I gobierno acaba de anunciar un proyecto de ley que crea el Fondo de Infraestructura
S.A. La propuesta, busca fomentar y expandir la inversión en infraestructura en Chile
contribuyendo a mejorar la productividad, la conectividad y la calidad de vida de las
personas'. En teoría, un incremento del $10 \%$ de la inversión en infraestructura pública, podría
traducirse en un alza de entre un 1,1\% y 2,3\% en el PIB (Calderón y Serven, 2014; Idrovo, 2012).
Actualmente, ya se habla de algunos ejes prioritarios para los próximos 10 años que incluyen
una infraestructura de sostén, logística y social (Cámara Chilena de la Construcción, 2016).
Sin embargo, llama la atención, la nula mención a la infraestructura científica-tecnológica
como elemento estratégico para las próximas décadas. A continuación, me centraré en la
importancia de invertir en nuevos espacios para el desarrollo de la I+D con el objeto de
avanzar hacia una productividad basada en la innovación.

(1) Proyecto de Ley que crea el Fondo de Infraestructura S.A. Información adicional en:

http://www.hacienda.cl/sala-de-prensa/infografias/proyecto-de-ley-que-crea-el-fondo-de.html

* Email de contacto: ljimenez@uahurtado.cl 
La importancia de la infraestructura en ciencia y tecnología

A diferencia de disciplinas como la administración, la economía o las humanidades, el desarrollo de la investigación científico-tecnológica requiere de espacios físicos especializados para que los investigadores puedan operar y responder a sus preguntas mediante la exploración de la naturaleza. En general, las preguntas que usa la Ciencia se denominan hipótesis de trabajo y el investigador las pone a prueba mediante un proceso conocido como desarrollo experimental. Hay actividades orientadas al descubrimiento y la exploración (ciencias básicas); mientras que otras, necesitan de una tecnología de apoyo para demostrar su funcionalidad (ciencias aplicadas).

Cualquiera sea el caso, el desarrollo científico requiere de una infraestructura física para dar soporte a nueva evidencia. En una primera etapa, el conocimiento emerge en forma de artículos y libros (derechos de autor). Luego, en una fase orientada a la aplicación, el conocimiento se protege como propiedad industrial (patentes y modelos de utilidad), para finalmente transferirse en productos y servicios que llegan al mercado (empresas).

\section{Tipos de Infraestructura}

Se reconocen distintos tipos de infraestructura que dan soporte a la investigación. Éstas van desde pequeños depósitos de almacenaje, hasta megaestructuras como son los observatorios astronómicos, laboratorios de seguridad, colecciones científicas, redes informáticas especializadas, buques de prospección, satélites, observatorios costeros, edificios y habitaciones inocuas o aisladas de la vibración, gravedad, ruido etc. Hay infraestructura tan costosa, que solamente puede concebirse mediante la cooperación internacional. Mientras que otras, han bajado dramáticamente sus costos de inversión como consecuencia de la madurez tecnológica de la disciplina. Ejemplo de ello son las infraestructuras del hardware computacional y redes informáticas, instrumentos de calibración, los insumos, servicios y reactivos de la biotecnología y análisis del genoma. También hay infraestructura que depende de su ubicación geográfica (radiotelescopios), del clima (soportes eólicos) y, por supuesto, de los fondos que permiten su operación y mantenimiento.

\section{La infraestructura científica como fuente de competitividad}

En la actualidad, el panorama global de la investigación científica es fluido, dinámico e intensamente competitivo. Los países desarrollados entienden que la inversión en infraestructura para la ciencia constituye un requisito clave para mantener sus capacidades de I+D y seguridad nacional. Históricamente, países como EEUU, Reino Unido y Canadá, construyeron su liderazgo tecnológico a partir de importantes inversiones en infraestructura. Con el tiempo, dichos espacios se transformaron en imanes para atraer al mejor capital humano y sirvieron de base para la colaboración internacional.

A diferencia de la infraestructura genérica como son los puentes, caminosy cárceles - que se deprecian por su usola infraestructura científica por definición crea valor. Su uso es proporcional al esfuerzo en I+D lo que se traduce en una mejora del desempeño tecnológico de la disciplina (innovaciones, patentes, spin-offs, licencias exclusivas). Hay una interesante rentabilidad en los proyectos de infraestructura científica que podrían atraer tanto a agentes públicos (rentabilidad social) como privados. La clave consiste en facilitar las relaciones ciencia-empresa e impulsar las actividades que fomentan la transferencia tecnológica y las estrategias de apropiabilidad² (Jiménez, 2016).

\section{El caso de Chile}

En Chile, las capacidades científicotecnológicas están circunscritas a las llamadas universidades tradicionales. Sus indicadores de productividad se reflejan principalmente en publicaciones académicas (libros y papers) que son reportadas anualmente por la Comisión Nacional de Investigación Científica y Tecnológica de Chile (CONICYT) ${ }^{3}$. De un total de 34 universidades adscritas al sistema único de admisión universitaria (DEMRE), solamente 5 instituciones son las que más publican en las áreas de la $\mathrm{OCDE}^{4}$. Los otros planteles tienen un impacto más bien discreto debido a su tamaño, orientación a la educación y/o trayectoria institucional. Menos evidente es la productividad de aquellas universidades llamadas de tiza y pizarrón, las cuales, al no contar con infraestructura científica, su impacto académico medido en $n^{\circ}$ de publicaciones y citas por paper - se ha visto fuertemente diezmada. Entre las razones que explican este hecho destacan: la baja frecuencia de publicaciones que exhiben las ciencias sociales y humanidades en comparación

(2) Todos los mecanismos para institucionalizar la aplicación y el respeto de la propiedad intelectual y sus contratos, incluida la facilidad para crear empresas (spin-off)

(3) http://www.conicyt.cl/blog/2016/02/productividad-de-universidades-cruch-segun-areas-ocde/

(4) Universidad de Chile (UCH), Universidad Católica (UC), Universidad de Concepción (UDEC), Universidad de Santiago (USACH) y Universidad Austral (UACH) 
a las ciencias exactas, las pocas revistas acreditadas y de calidad en la disciplina, y el bajo apoyo que otorgan las agencias gubernamentales y privadas al desarrollo de las ciencias sociales. A modo de contraste, un laboratorio de bioquímica conformado por 2 doctores y 4 asistentes de investigación puede llegar a publicar en promedio 5 artículos Web of Science anualmente ${ }^{5}$.

\section{La infraestructura científica de ayer y de hoy}

Por años, las universidades tradicionales construyeron sus capacidades gradualmente, migrando desde el naturalismo científico hacia disciplinas altamente especializadas y diversas. Por ejemplo, la ciencia de la morfología donde antes predominaban las lupas y esqueletos - fue cambiada por el paradigma de la biología molecular. También eran otros los precios de la tecnología. Hasta hace 25 años, los equipos de base de la biología experimental eran mucho más caros y gozaban del monopolio legal que le conferían sus patentes de invención. Por lo tanto, no existía la posibilidad de encontrar sustitutos, ni una cadena de proveedores establecida. Tampoco operaban los tratados de libre comercio, por lo que las ventajas arancelarias que favorecen la importación de la tecnología eran recién un proyecto.

En la actualidad, para acceder a equipamiento institucional, las universidades -incluidos los centros de investigación públicos- participan de un conjunto de fondos concursables distribuidos entre los Ministerios de Educación (MINEDUC), Economía
(MINECON) y Agricultura (MINAGRI), los cuales operan el presupuesto a través de agencias ejecutoras como son Conicyt, Innova Chile (de CORFO), la Iniciativa Científica Milenio (ICM) y la Fundación para la Innovación Agraria (FIA). Desde el retorno a la democracia, ha sido el Conicyt, mediante el Fondo de Fomento al Desarrollo Científico y Tecnológico (Fondef) y el Fondo Nacional de Desarrollo Científico y Tecnológico (Fondecyt), la agencia relevante que ha contribuido a sostener la infraestructura científica y tecnológica de Chile.

Otras iniciativas, como el Programa de Investigación Asociativa (PIA) creado en el 2009-y el Fondo para el Equipamiento Científico y Tecnológico (Fondequip) — creado en el 2011también han contribuido notablemente al fomento de la investigación asociativa y al financiamiento de apoyo a la infraestructura. Según el Conicyt (en base a DIPRES, 2012), el déficit en equipamiento en I+D para el 2010 estaba estimado en unos \$MM 28.600. Algo de esa brecha, ya ha sido cubierta en parte por el Fondequip con un financiamiento acumulado de unos \$MM 22.500 al $2015^{6}$. No obstante, los \$MM 6.000 faltantes, el rezago tecnológico entre el año 2010 y el presente, la nueva demanda por el uso de laboratorios y las tasas de depreciación tecnológica, hacen presumir que las necesidades de infraestructura actuales son, por lejos, mucho mayor a las estimadas originalmente.

\section{Algunas propuestas y desafíos}

Es claro, que la infraestructura en ciencia y tecnología es fundamental para el desarrollo de las actividades de I+D. Hoy en día, los académicos realizan principalmente dos tipos de actividades: investigación y formación de pre y posgrado. Ambas funciones requieren de un uso intensivo de los equipos de laboratorio, insumos y espacios físicos para trabajar. En la práctica, la mezcla de ambas actividades produce una subvaloración importante en los proyectos de I+D, pues los estudiantes - para bendición de muchos - son los que más usan y deprecian la infraestructura con sus actividades de formación. Lo anterior, se traduce en espacios muy demandados, en donde pululan los investigadores, tesistas y becarios de postgrado que buscan un lugar para insertarse laboralmente. Se estima que el número de doctorados podría aumentar en un $88 \%$ - respecto del 2012-lo que implicaría aproximadamente unos 4.000 nuevos investigadores para el 2018 (González y Jiménez, 2014).

Otro déficit importante, es la falta de infraestructura para escalar la investigación básica hacia el desarrollo y la innovación. En Chile, casi todo el financiamiento público para la investigación está orientado a instituciones sin fines de lucro. Esto plantea importantes desafíos legales y de gestión institucional. No es fácil desarrollar en las universidades actividades de fomento a la innovación que impliquen negocios en asociación con empresas, pues no existen normativas que regulen la propiedad de los resultados de la I+D. Tampoco hay esquemas de reparto de beneficios que consideren a los académicos y sus laboratorios ${ }^{7}$. A pesar de lo anterior,

(5) Después de astronomía y astrofísica, la bioquímica y biología molecular es el área que más publica en Chile, con una producción acumulada de 1675 artículos entre el 2000 y el 2015.

(6) El cálculo se realizó en base a unos gráficos presentados por María Elena Boisier Pons, Presidenta (S), CONICYT, 14 de abril de 2014 + la lista de proyectos financiados por el fondequip 2015 disponibles en http://www.conicyt.cl/fondequip/ 
algunas incubadoras han realizado importantes esfuerzos por apoyar emprendimientos de base científica. Lamentablemente, no sirven de mucho los servicios valorados de gestión y vinculación a redes, si no existe una infraestructura que soporte el desarrollo experimental que requiere un producto antes del llegar al mercado.

En consecuencia, es clave avanzar hacia una infraestructura distinta y separada de la institucionalidad pública de la Universidad. Así, se haría más fácil la productividad basada en innovación, el fomento de las relaciones cienciaempresa y la generación de negocios de base científica (Jiménez, 2016).

\section{Conclusión}

El proyecto de infraestructura impulsado por el gobierno es una interesante oportunidad para poner en debate, la importancia estratégica de construir en Chile una plataforma para el desarrollo científico-tecnológico. Hay una carencia notable de infraestructura en las universidades que podría afectar no solamente la calidad de la investigación, sino también, las actividades educacionales y formativas que están íntimamente ligadas al uso de los laboratorios. Actualmente, la reforma educacional impulsada por el Gobierno se olvida que la investigación y la educación son actividades ligadas a una infraestructura especializada, distinta a las salas de clases. Por ende, es clave para una educación de calidad, renovar, sostener e invertir en nuevos laboratorios para la Ciencia. No es lo mismo una formación profesional vinculada a modernos equipos, que una educación con una infraestructura científica obsoleta y desactualizada. Lo anterior se hace evidente en aquellas universidades con carreras científicotecnológicas y que albergan disciplinas con eminente potencial innovador.

También faltan espacios para escalar las ciencias básicas hacia productos y servicios con valor de mercado. No siempre es posible usar la misma infraestructura de la Universidad para la exploración de negocios surgidos de la I+D. En un entorno cada vez más público de la educación universitaria chilena, no es prudente mezclar ambas actividades. El actual proyecto de la educación superior es claro y se contemplan penas de cárcel para el delito de apropiación indebida. Esto implica, que cualquier acción legal y/o malintencionada, podría tener espacio para objetar los intentos de investigadores por hacerse parte de activos surgidos de un financiamiento estatal. Este asunto debe ser tratado con la mayor diligencia y seriedad por los planteles educacionales. El Gobierno, por su parte, debe hacer explícitos sus esfuerzos para clarificar sus reformas e impulsar el escalamiento de la ciencia hacia el desarrollo y la innovación. No es posible, que una reforma orientada a mejorar la educación superior, termine entrampando el derecho a emprender e innovar a partir de los resultados de la I+D.

Finalmente, las universidades de tiza y pizarrón - que surgieron en Chile con motivaciones fundacionales distintas al desarrollo tecnológicopodrían reconsiderar la inclusión de las ciencias experimentales en su foco estratégico de crecimiento. Se elevaría notablemente su base de publicaciones y les permitiría acceder a una amplia diversidad de fondos de desarrollo científico-tecnológico. Además, los costos de inversión y mantención de laboratorios se han vuelto muy competitivos en el último tiempo, lo que podría abrir interesantes oportunidades de expansión y desarrollo institucional.

\section{Referencias}

Calderón, César and Servén, Luis, Infrastructure, Growth, and Inequality: An Overview (September 1, 2014). World Bank Policy Research Working Paper No. 7034. Available at SSRN: http://ssrn.com/abstract=2497234 Idrovo Aguirre, Byron (2012): Inversión en infraestructura pública y crecimiento económico, evidencia para Chile. Published in:Documentos deTrabajo delaCámaraChilena delaConstrucción No. No. 69 (1. March 2012)

Jiménez, A. (2016). Relaciones universidad-empresa: Hacia una productividad basada en innovación. Gestión Y Tendencias, 2(1), 7-10. doi:http://dx.doi.org/10.11565/ gesten.v2i1.11

Gonzalez, H., \& Jiménez, A. (2014). Inserción Laboral de Nuevos Investigadores con Grado de Doctor en Chile. Journal of Technology Management \& Innovation, 9(4), 132148. doi:http://dx.doi.org/10.4067/ S0718-27242014000400010

(7) También hay elementos en la propia ley chilena que impiden que un funcionario público se apropie de activos que surgen de un financiamiento estatal. Ejemplo de ello es el Estatuto Administrativo o la Ley de Probidad, que regula el régimen del Funcionario Público, y que podrían impedir que los académicos de universidades estatales realicen actividades emprendedoras dentro de la Universidad sobre ciertos montos bastante bajos para este ámbito (200 UTM). Además, las direcciones jurídicas, con respaldo de la Contraloría General de la República, han establecido un marco estricto y algo desmotivante para las actuaciones de académicos que destinan tiempo en actividades como los spin offs. Incluso podría representar un conflicto de intereses por la incompatibilidad legal que implica desarrollar actividades propias de un funcionario público por una parte y otras que buscan apropiarse y/o explotar comercialmente activos que surgen de un financiamiento estatal. 